\title{
Variability of the Doppler gradient in pulmonary valve stenosis before and after balloon dilatation
}

\author{
M K LIM,* A B HOUSTON, W B DOIG, S LILLEY, E P MURTAGH \\ From the Department of Cardiology and Department of Child Health, Royal Hospital for Sick Children, \\ Glasgow
}

SUMMARY The variability of the valve gradient measured by Doppler in pulmonary stenosis was compared with the variability of the gradient measured at catheterisation in 42 infants and children undergoing catheterisation with a view to balloon dilatation of the pulmonary valve. The maximum value measured by Doppler when the patient was unsedated was significantly higher than that measured when the patient was sedated for catheterisation, and the maximum gradient was significantly higher shortly after than several days later. In a patient with pronounced infundibular obstruction after dilatation the Doppler signal clearly showed that the obstruction was dynamic, with a superimposed lower fixed signal that correctly predicted the final low gradient.

The Doppler gradient in an alert and unsedated patient may be a better measure of the true physiological value. The highest Doppler value so obtained is a more appropriate indicator of the need for balloon dilatation than a single catheter measurement. The result of dilatation is best assessed by Doppler measurement at least a day after the procedure.

Doppler echocardiography accurately estimates the severity of pulmonary valve stenosis, ${ }^{1-3}$ and the agreement with the results of catheterisation is best when both measurements are performed simultaneously. ${ }^{34}$ This indicates that gradients vary under different physiological conditions. It has been generally accepted, but not confirmed, that the gradient in an alert and possibly apprehensive patient can be higher than that measured when the patient is sedated at catheterisation.

Decisions on the need for intervention have previously been based on the transvalvar gradient measured at catheterisation in sedated patients. But the values of the Doppler gradient may be different in an alert patient, and this might influence decisions on the need for or results of dilatation.

We report our findings with Doppler ultrasound in 42 patients with pulmonary valve stenosis undergoing catheterisation to establish whether they needed balloon dilatation. We wanted to see whether the measured gradient was different in sedated patients

Requests for reprints to Dr A B Houston, Royal Hospital for Sick Children, Yorkhill, Glasgow G3 8SJ.

^Present address: Paediatric Department, General Hospital, Kuala Lumpur, Malaysia.

Accepted for publication 14 March 1989 and whether it changed between the period immediately after dilatation (under sedation) and later follow up.

\section{Patients and methods}

A series of 42 patients aged six days to 15 years (mean 4 years 10 months) underwent cardiac catheterisation to assess their suitability for balloon dilatation of the pulmonary valve. In six patients dilatation was not undertaken because the physician considered that the gradient was not clinically significant. Dilatation was attempted in the remaining 36: one infant with critical pulmonary stenosis became hypotensive when the guide catheter traversed the pulmonary orifice, and it subsequently proved impossible to pass the balloon catheter into the pulmonary artery; the gradient seemed little changed by the procedure in five patients and reduced in the remaining 30 .

Catheterisation was performed under sedation with either a mixture of pethidine, promethazine, and chlorpromazine as a single intramuscular injection or oral phenobarbitone followed by a single intramuscular dose of papaveretum and hyoscine. Right ventricular and pulmonary arterial pressures were measured through fluid filled catheters attached to strain gauge transducers either simultaneously through two catheters or by withdrawal across the valve. 


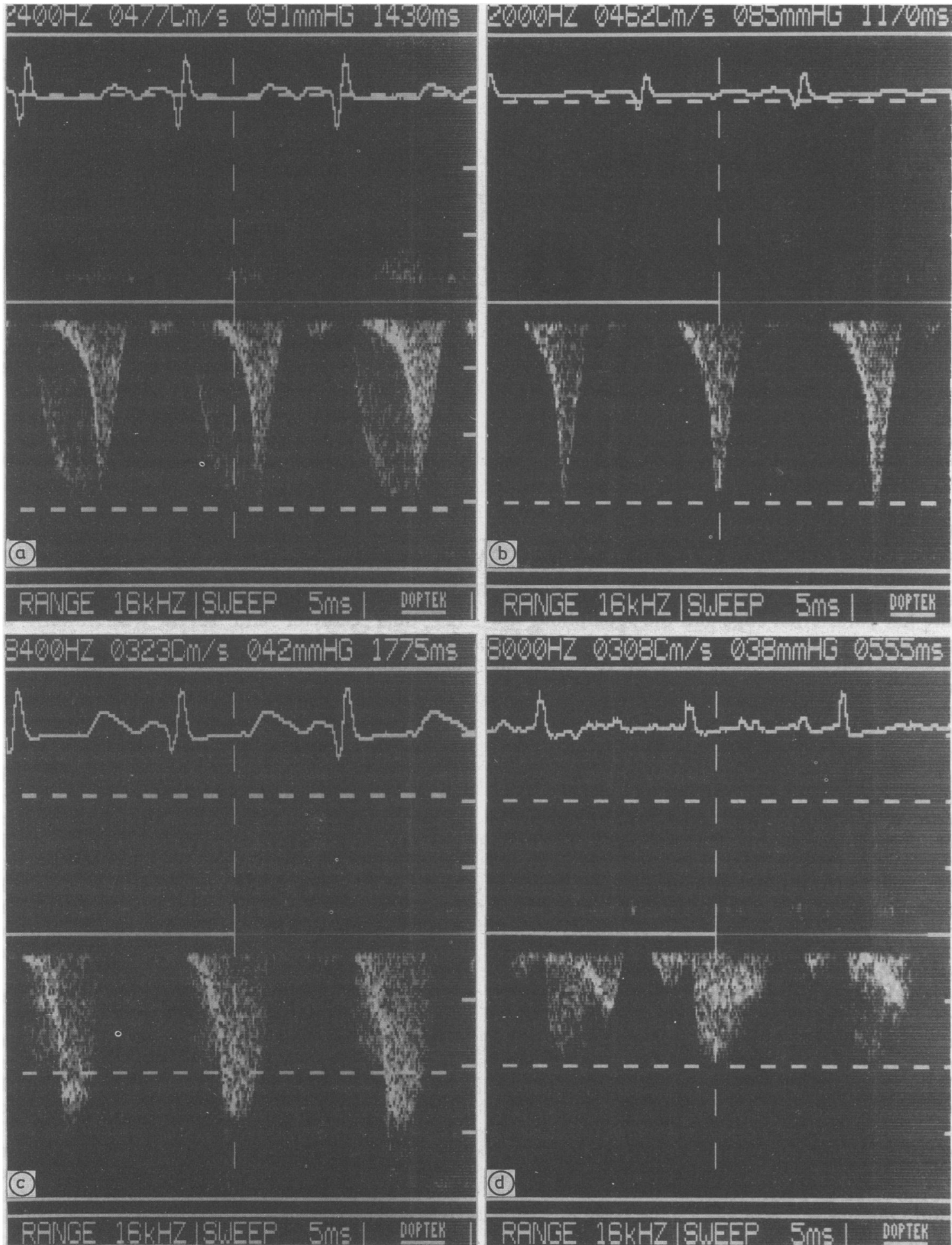

Fig 1 Spectral recordings from a three month old patient with pulmonary valve stenosis and significant subvalvar dynamic muscular obstruction. Before dilatation (a) the dynamic concave signal (equivalent to a gradient of $85 \mathrm{~mm} \mathrm{Hg}$ ) was superimposed on the signal indicating fixed obstruction ( $91 \mathrm{~mm} \mathrm{Hg})$, but (b) was shown more clearly by adjustment of transducer angulation. Immediately after (c) the dynamic concave signal ( $85 \mathrm{~mm} \mathrm{Hg}$ ) remained but the fixed one was reduced to $42 \mathrm{~mm} \mathrm{Hg}$, but (d) 6 weeks later the gradient was $38 \mathrm{~mm} \mathrm{Hg}$ with a low dynamic signal, equivalent to $12 \mathrm{~mm} \mathrm{Hg}$. 


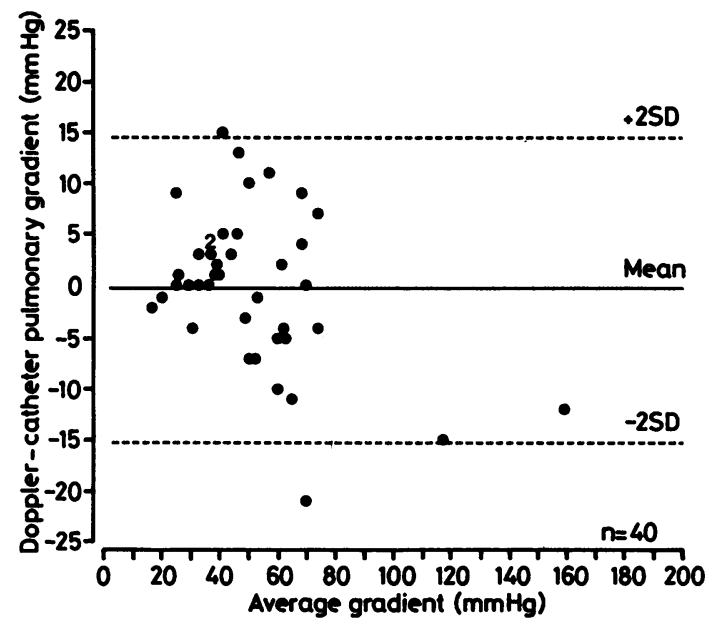

Fig 2 Comparison of the difference between gradients (Doppler minus peak to peak) and the average gradient obtained at the time of catheterisation before balloon dilatation in 40 patients.

Doppler studies were performed with either a nonimaging Alfred Velocimeter (Vingmed) interfaced to a Doptek spectrum analyser or a Vingmed CFM 700 system with imaging and colour flow mapping. The entire left parasternal edge and the subxiphoid and suprasternal positions were explored and the position and angulation of the probe adjusted to obtain the maximum reproducible velocity signal showing the high velocity signals with a clear and complete envelope. Simultaneous electrocardiographic monitoring was used to exclude maximal frequency shifts caused by extrasystoles. The maximum frequency shift was measured from the Doppler signal and the maximum velocity $(V)$ was derived and the pressure drop ( $P$ ) estimated from the modified Bernoulli formula, $P=4 V^{2}$.

For further confirmation of the accuracy of our technique we compared Doppler values with simultaneous peak to peak pressure gradients before dilatation was attempted and just before the catheters were removed after dilatation. The difference between the values was calculated, the mean difference (and standard deviation) for the studies obtained, and the individual differences were plotted against the average values by the method of Bland and Altman."

Then the highest Doppler estimate of the pressure drop obtained without sedation was compared with the value when the patient was sedated at catheterisation before dilatation, and the Doppler measurement 15 to 45 minutes after the procedure was compared with the value $\geqslant 1$ days later.

Full Doppler and catheter studies were not available for review from every patient: Doppler gradients

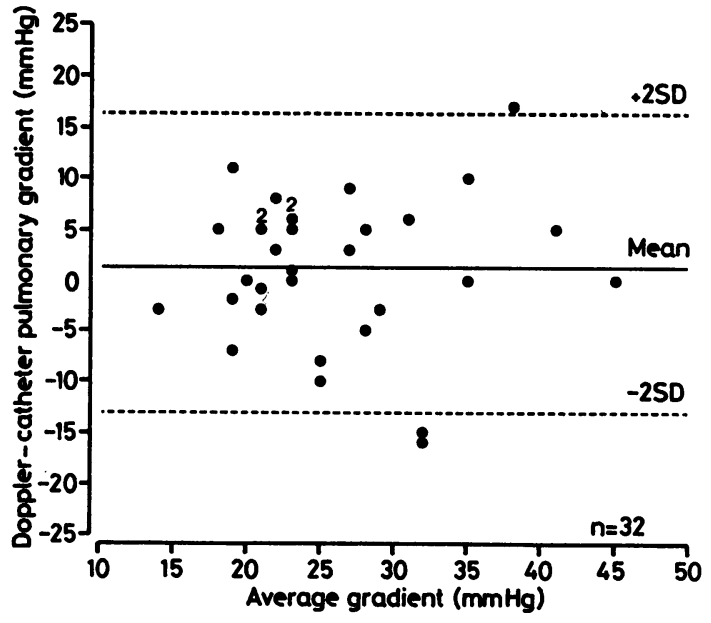

Fig 3 Comparison of the difference between gradients (Doppler minus peak to peak) and the average gradient obtained at the time of catheterisation after balloon dilatation in 32 patients.

measured with and without sedation were available in 38; simultaneous Doppler and catheter gradients before dilatation in 40 (29 dual catheter and 11 withdrawal) and immediately after dilatation in 32 (25 dual catheter and seven withdrawal); and Doppler gradients immediately and $\geqslant 1$ days after the procedure in 30 .

\section{Results}

\section{DOPPLER FLOW PATTERNS}

When patients were under sedation before dilatation all the Doppler records showed the typical parabolic appearance of fixed obstruction, with a lower velocity concave (or scimitar) signal of dynamic obstruction clearly superimposed in six. The dynamic obstruction was relatively unimportant being $<20 \mathrm{~mm} \mathrm{Hg}$ in all but two. In one it was $100 \mathrm{~mm} \mathrm{Hg}$ for both the fixed and dynamic gradient and in the other patient the dynamic obstruction was $85 \mathrm{~mm} \mathrm{Hg}$ and the fixed obstruction was $91 \mathrm{~mm} \mathrm{Hg}$ (fig 1 a and b). In this last patient the fixed element was $42 \mathrm{~mm} \mathrm{Hg}$ and the dynamic element was $85 \mathrm{~mm} \mathrm{Hg}$ immediately after dilatation (fig 1c); single catheter measurements showed a total pressure drop of $130 \mathrm{~mm} \mathrm{Hg}$ between the pulmonary artery and ventricle $-40 \mathrm{~mm} \mathrm{Hg}$ from the pulmonary artery to a subvalve chamber and $90 \mathrm{~mm} \mathrm{Hg}$ from there to the ventricle. The spectral signal was interpreted as indicating that the valve narrowing was sufficiently reduced but there was still appreciable dynamic infundibular obstruction, and we predicted that the dynamic obstruction would resolve leaving a final gradient of about 


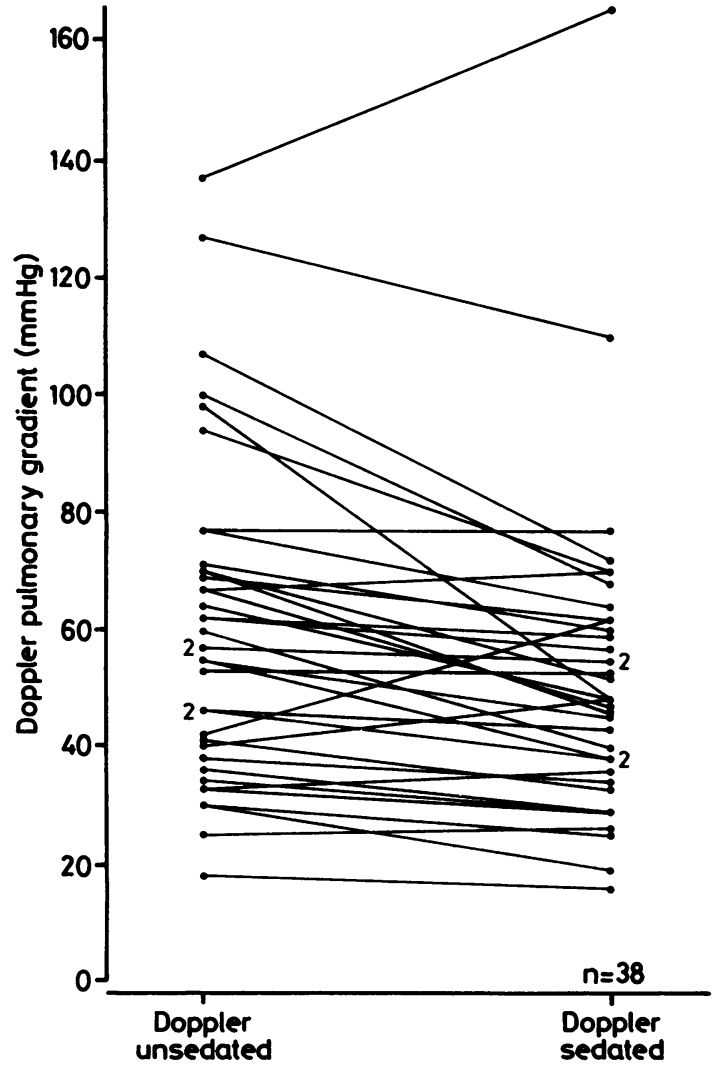

Fig 4 Differences between maximum Doppler gradient obtained in unsedated patients before balloon dilatation and that measured at catheterisation in 38 sedated patients.

$40 \mathrm{~mm} \mathrm{Hg}$; four months later the Doppler gradient was $38 \mathrm{~mm} \mathrm{Hg}$ with only a minor dynamic component $(12 \mathrm{~mm} \mathrm{Hg})$ (fig $1 \mathrm{~d})$.

\section{COMPARISON OF DOPPLER VALUES WITH PEAK}

TO PEAK MAXIMUM GRADIENT

Comparison of these by the method of Bland and Altman ${ }^{5}$ gave the mean difference (2 SD) between methods before dilatation of $-0.1(15.0) \mathrm{mm} \mathrm{Hg}$ (fig 2) and of 1.2 (14.6) $\mathrm{mm} \mathrm{Hg}$ after dilatation (fig 3).

\section{COMPARISON OF DOPPLER VALUES IN THE}

\section{SEDATED AND UNSEDATED STATE}

The maximum values for the Doppler gradients with the patient unsedated were compared with those measured under sedation in 38 patients (fig 4) by a paired $t$ test on the differences between values measured with and without sedation. The mean difference between sedated and unsedated values (2 SD) $(8.2(3.7) \mathrm{mm} \mathrm{Hg}$ ) was statistically significant $(p<0.01)$. In general the values in unsedated

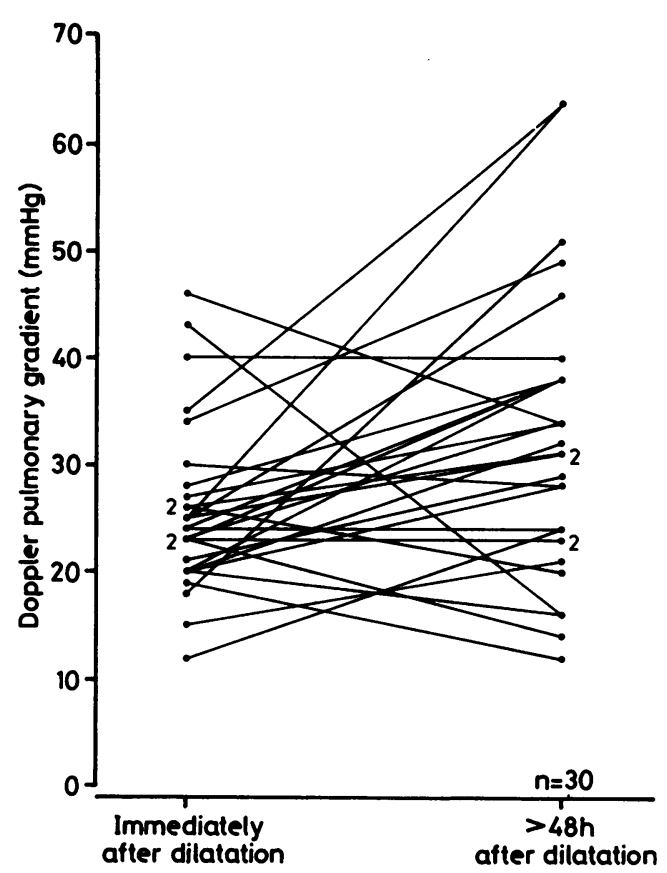

Fig 5 Doppler gradient measured in 30 sedated patients immediately after balloon dilatation and the value at least two days later.

patients were higher. In 22 patients the values without sedation were within $10 \mathrm{~mm} \mathrm{Hg}$ of the values measured under sedation, in 14 cases the value in the unsedated patient was $>10 \mathrm{~mm} \mathrm{Hg}$ higher, and in only two patients was it $>10 \mathrm{~mm} \mathrm{Hg}$ less.

\section{COMPARISON OF DOPPLER VALUES}

IMMEDIATELY AND > 1 DAY AFTER DILATATION The patient with considerable residual infundibular obstruction (see below) was excluded. In the remaining 30 patients (fig 5) a two tailed paired $t$ test on the differences between Doppler values shortly after dilatation and $>24$ hours after was significant ( $p<$ 0.01 ) (mean difference (2 SD), 6.8 (5.0) $\mathrm{mm} \mathrm{Hg}$ ). These results show that the values obtained immediately after dilatation were significantly lower than those 24 hours after the procedure. The changes in Doppler values were $>10 \mathrm{~mm} \mathrm{Hg}$ in 11 cases. The greatest difference was in a patient who had a pulmonary valve gradient of $35 \mathrm{~mm} \mathrm{Hg}$ by Doppler (measured as $35 \mathrm{~mm} \mathrm{Hg}$ peak to peak) shortly after dilatation, but which rose to $64 \mathrm{~mm} \mathrm{Hg} 48$ hours after the procedure.

\section{Discussion}

Doppler ultrasound accurately measures the transvalvar gradient in pulmonary valve stenosis, ${ }^{1-4}$ and 
the decision on whether to proceed to dilatation is now based on a Doppler gradient obtained when the patient is active, usually at an outpatient visit, rather than on catheter measurements of pressure under sedation. It is essential to have clear knowledge of possible changes in gradient that can result from this.

This study confirmed the accuracy of Doppler in comparison with simultaneous catheter readings but showed that the gradient usually fell with sedation: in most cases this fall was small $(<10 \mathrm{~mm} \mathrm{Hg}$ ) and of little clinical significance but in some it can be so large that the gradient is no longer considered to be of clinical significance. Is the gradient measured under sedation an appropriate one on which to base the decision on the need for dilatation? We believe that it is more logical to use the highest value, even if this is obtained in an unsedated patient, because this will more accurately reflect the true physiological condition.

Robertson et al found no significant alteration in gradients measured immediately after dilatation and several days later but gave only the mean of the gradients in a group of 18 patients. ${ }^{6}$ Our study indicates that changes do occur, as shown by the $t$ test, although in only two of the 30 was this change of clinical significance.

We found a statistically significant difference between the Doppler gradients measured in the active and the sedated states before and after dilatation, and it is important to consider these changes when patients are selected for dilatation or the results of this procedure are assessed. We consider the highest gradient, whether measured in a patient who is awake or sedated, is the most appropriate for selecting patients for dilatation and that a repeat Doppler study several days thereafter is essential in assessing the final result.

\section{References}

1 Lima CO, Sahn DJ, Valdes-Cruz LM, et al. Noninvasive prediction of transvalvular pressure gradient in patients with pulmonary stenosis by quantitative two-dimensional echocardiographic Doppler studies. Circulation 1983;67:866-71.

2 Johnson GL, Kwan OL, Handshoe S, Noonan JA, De Maria AN. Accuracy of combined two-dimensional echocardiography and continuous wave Doppler recordings in the estimation of pressure gradient in right ventricular outlet obstruction. J Am Coll Cardiol 1984;3:1013-8.

3 Houston AB, Sheldon CS, Simpson IA, Doig WB, Coleman EN. The severity of pulmonary valve or artery obstruction in children estimated by Doppler ultrasound. Eur Heart J 1985;6:786-90.

4 Currie PJ, Hagler DJ, Seward JB, et al. Instantaneous pressure gradient: a simultaneous Doppler and dual catheter correlative study. J Am Coll Cardiol 1986; 7:800-6.

5 Bland JM, Altman DG. Statistical methods for assessing agreement between two methods of clinical measurement. Lancet 1986;i:307-10.

6 Robertson M, Benson LN, Smallhorn JS, et al. The morphology of the right ventricular outflow tract after percutaneous pulmonary valvotomy: long term follow up. Br Heart J 1987;58:239-44. 